Horizons philosophiques

\title{
Husserl et l'absolu du Monde en phénoménologie
}

\section{Éric Paquette}

Volume 9, numéro 1, automne 1998

\section{Médiations}

URI : https://id.erudit.org/iderudit/801091ar

DOI : https://doi.org/10.7202/801091ar

Aller au sommaire du numéro

\section{Éditeur(s)}

Collège Édouard-Montpetit

\section{ISSN}

1181-9227 (imprimé)

1920-2954 (numérique)

Découvrir la revue

Citer cet article

Paquette, É. (1998). Husserl et l'absolu du Monde en phénoménologie. Horizons philosophiques, 9(1), 51-71. https://doi.org/10.7202/801091ar d'utilisation que vous pouvez consulter en ligne.

https://apropos.erudit.org/fr/usagers/politique-dutilisation/ 


\section{HUSSERL ET L'ABSOLU DU MONDE EN PHÉNOMÉNOLOGIE}

\section{Verse et controverse sur l'opposition idéalisme/réalisme}

Qu'on le veuille ou non, il n'appert plus possible d'échapper à la nécessité de prendre position dans une "polémique" devenue, à tort ou à raison, le centre d'un enjeu désormais incontournable : l'alternative réalisme/idéalisme dans l'interprétation de la théorie husserlienne de la constitution du Monde par le Sujet transcendantal.

Plusieurs dénoncent l'inanité heuristique d'une opposition si faiblement "phénoménologique». Pour certains, les termes mêmes du débat - on ne peut plus traditionnels - auraient déjà perdu toute raison d'être par suite du nouvel esprit de la philosophie phénoménologique transcendantale. Pour d'autres, l'une des options traditionnelles s'en trouverait nécessairement et unilatéralement condamnée : ou bien l'on qualifie l'idéalisme d'absolument contraire à l'intuitionnisme phénoménologique, ou bien le réalisme d'absolument incompatible avec l'activité constituante de l'Ego. Pour d'autres encore, l'évolution de la pensée husserlienne comporterait un tournant à cent quatrevingts degrés, ou moins, selon le point de vue : d'abord réaliste, Husserl se serait ensuite tourné vers l'idéalisme (d'aucuns présentent encore les Ideen I comme pivot de cette conversion, ou dérive, toujours selon le point de vue).

II est certes possible, et même vraisemblable, que le débat réalisme/idéalisme, appliqué à la phénoménologie, relève d'une projection métaphysique stérile, sinon d'un gigantesque malentendu. Pour peu que l'antonymie réalisme-idéalisme ait une prétention épistémologique universelle, sa tension eidétique fondamentale doit s'inscrire au niveau dernier des catégories de la représentation onto-logique. Tiré hors du cadre métaphysique où la tradition lui donna forme (donc sens), le couple réalisme/idéalisme se voit dénaturé en vaine dualité, avant que 
de périr en un vain «duel». Sur le terrain de la «positivité», les oppositions fondationnelles se peuvent exprimer moins radicalement, moins dangereusement : sans le péril de l'«absolutisme». À toutes fins utiles, les épistémologies régionales n'ont jamais besoin d'absolutiser - non plus que de nier- la part "créatrice» de la "subjectivité» (de l'entendement) dans la genèse historico-transcendantale du Savoir.

Tous les chantres de l'Absolu (métaphysiciens, théosophes \& mystiques confondus) auront beau nous promettent la fusion des opposés dans l'Absolu à venir (ou l'Avenir absolu)... soit! mais nous n'en sommes pas là. Chacun doit à présent souffrir la division des langues et la multitude des signes pour se déchiffrer soi-même et deviner autrui. L'autologie de l'Identité absolue abolit l'identité "réelle» de la logique multiple "du réel»; voire des logiques (même en logique le pluriel s'impatronise aujourd'hui). II n'est point de pensée logique, ni de pensées tout court, sans différenciations, sans limitations. Omnis determinatio est negatio : l'adage spinoziste ne vieillit point, il se perpétue et se répand. La défiance de la phénoménologie face aux "oppositions" trop lourdement spéculatives n'est pas étrangère à ce souci de la nuance transcendantale qu'est l'attitude de réduction. Les oppositions immodérées finissent bien souvent par s'invertir à force de démesure. II est un sens, connu et reconnu par Husserl, où le subjectivisme tourne en objectivisme. Tel est le "paradoxe" d'une rationalité qui use et abuse d'elle-même par excès de métaphysique. Se référant aux grandes oppositions léguées par la tradition (rationalisme versus empirisme, interprétation théologique du Monde versus interprétation causaliste, subjectivisme versus objectivisme, ontologisme versus transcendantalisme, psychologisme versus antipsychologisme, positivisme versus métaphysique), Husserl y va de cette remarque : "Chaque thèse est fondée en raison, mais chacune est imparfaite en elle-même, ou bien absolutise un point de vue unilatéral relatif et abstrait 1 ».

1. "La phénoménologie (article pour l'Encyclopaedia Britannica), première version" in Notes sur Heidegger, traduit par Didier Franck, Paris : Minuit, 1993, p. 91. 
Que le dessein d'approcher le concept de constitution par le biais du débat idéalisme/réalisme emprunte une voie bien hasardeuse, cela ne fait aucun doute. Mais saurait-on vraiment échapper au gigantesque labyrinthe du langage déjà constitué? Husserl lui-même n'a pu trouver la sortie, se voyant forcé de ressasser l'ancien lexique pour faire valoir un sens tout neuf. D'où l'ambivalence de son "idéalisme" transcendantal" et du concept de constitution qui le caractérise. Étant donné l'incessant remaniement de la Konstitutionslehre, les hésitations à peine voilées de son fondateur, l'appréciation mitigée de Fink (ce témoin par excellence, jouissant de l'intimité amicale du "Maître de Fribourg"), l'existence d'une respectable et féconde tradition de phénoménologues "constructivistes", de Cassirer à Marc Richir, etc., on ne peut que reconnaître la légitime pluralité des interprétations. En pareil cas, personne n'a sans doute absolument raison, ni personne absolument tort. Ce qui compte alors, ce nous semble, n'est pas tant la prétention des uns et des autres à la vérité de l'intention husserlienne, que la possibilité pour chacun de suivre une piste d'interprétation légitime tout en reconnaissant ses présupposés.

\section{La Transcendance du Monde}

Aussi vrai que la théorie de l'intuition soit le cœur de la philosophie husserlienne, l'analyse de la synthèse passive nous paraît le centre et le terme de la phénoménologie génétique et constitutive. Suivant cette optique : la théorie de la constitution doit forcément répondre du principe de la donation originelle, du primat de l'intuition sur toute construction ; elle doit pouvoir justifier la double affirmation de la Transcendance du Monde et de son idéalité transcendantale. Telle est l'assurance que partagent dans leur ensemble les interprètes "anti-constructivistes» de la constitution, dont nous sommes. Avant d'étayer ce principe d'interprétation, il faut d'abord s'assurer soi-même, en se fondant sur la lettre husserlienne que la phénoménologie reconnaît bel et bien et absolument l'absolu du Monde. À cette fin, qu'on nous permette ici d'égrener un long chapelet de citations probantes, pour ne pas dire concluantes. 
Dès l'hiver 1910-1911, dans ses leçons sur les Problèmes fondamentaux de la phénoménologie, Husserl appréhende très clairement la signification normative-transcendantale du concept naturel de Monde, et par là, la possibilité d'une fondation ou justification transcendantale de l'opposition Moi/Monde. II s'oppose alors de plein fouet à l' «empiriocriticisme" d'Avenarius, mais aussi, forcément, à toute philosophie qui finirait par rendre absurde la différence ontologique entre le Moi et le Monde. Quand bien même la référence onto-logique du Moi au Monde ne serait qu'une "façon de parler", il nous en faudrait toujours reconnaître la nécessité : «aussi longtemps, riposte Husserl, que parler d'un monde où sont les hommes, lesquels ont conscience du monde, ont des vécus, et, parmi eux, ont des perceptions, des expériences, etc., posant de l'être-là empirique, aussi longtemps que parler ainsi conserve son sens, aussi longtemps vaut absolument et a priori le concept naturel de monde 2 ". Sur le même thème, l'appendice xxi (Philosophie immanente. Avenarius), probablement rédigé en 1915, reconnaît comme "précompréhension" de toute compréhension, le "don" originel du monde : "Avant toute théorie, le monde est donné. Toutes les visées, légitimes ou illégitimes, populaires, superstitieuses, scientifiques, toutes se rapportent au monde déjà donné d'avance ${ }^{3}$ ».

La phénoménologie et les fondements des sciences («Manuscrit au crayon», 1912) poursuit l'analytique transcendantale à travers une épistémologie résolument «intuitionniste», soucieuse d'obéir au principe des principes, au primat du donné et de la donation, à l'autorité de l'intuition, c'est donc dire à la norme de l'«expérience». Or, il n'est point de réelle expérience sans (re)connaissance d'un expérimenté : «En toute sphère de la réalité, écrit Husserl, il y a description et la description constate l'être-là, l'être-là réel. Cela revient à dire : toute description exprime à l'aide de mots et de significations de mots

2. Problèmes fondamentaux de la phénoménologie, traduit par Jacques English, Paris : P.U.F., 1991, § 10, p. 124 [136].

3. Ibid., p. 219 [196]. 
ce qui est expérimenté 4[...]" Le corps des sciences, cherchant sans cesse à préciser le sens concret, le contenu, de la Thèse du monde, présuppose toujours l'ordre objectif (et «objectal») de la Nature, à commencer par l' "autonomie" du monde matériel : "le monde matériel est à l'intérieur du monde objectif total que nous appelons nature, un monde propre et fermé sur soi, un monde qui n'a besoin du secours d'aucune autre réalité5».

Les Ideen I (1913), dont l'approche «ego-centrique» de la réduction (dite plus tard "chemin cartésien») sembla consacrer le passage à l'idéalisme, ne laissaient pourtant aucun doute sur la valeur inaliénable de la croyance au monde. La réduction n'est pas le doute, moins encore la dénégation. Au dire même de Husserl, la réduction, en sa forme primordiale, l'épochè phénoménologique, n'a d'intérêt qu'en vue d'une Aufhebung de la Thèse du monde. "Non point, précise-t-il, que la thèse se convertisse en antithèse, la position en négation; ou qu'elle se change en conjecture, supputation, indécision, doute (quel que soit le sens du mot); rien de tout cela n'est au pouvoir de notre libre arbitre 6". L'opération de réduction préside à la "suspension", "mise en suspens", ou "mise entre parenthèses", d'une certaine "position d'existence» (ou «thèse"). En sa généralisation universelle, elle permet de concevoir (= constituer le concept de) la possibilité logique de la non-existence ou noneffectivité du Monde. Le "doute" qui voudrait sourdre de cette possibilité n'est pas un doute véritable, mais une pure «fiction" (transcendantale). En tant qu'intention invariablement vide, ce pur possible logique nous révèle à nous-mêmes dans notre propre impossibilité d'y consentir. Voilà pourquoi Fink a pu décrire l'épochè phénoménologique comme une "authentique découverte de la croyance au monde, la découverte du monde comme dogme transcendantal $7[$...]".

4. La phénoménologie et les fondements des sciences, traduit par Dorian Tiffeneau, Paris : P.U.F., 1993, p. 55 [45].

5. Ibid., p. 139 [117].

6. Idées I, traduit par Paul Ricoeur, Paris : Gallimard, 1985, § 31, p. 98 [54] (c'est nous qui soulignons).

7. “La philosophie phénoménologique d'Edmund Husserl face à la critique contemporaine" in De la phénoménologie, Paris : Minuit, 1974, p. 135. 
II nous paraît très significatif de noter que la préface à l'édition anglaise des Ideen I, rédigée par Husserl en 1930, et dont l'objectif fut précisément de dissiper les plus graves malentendus touchant la phénoménologie, porte quasi exclusivement sur la confrontation idéalisme/réalisme. Husserl y formule en ces termes la différence essentielle entre son «idéalisme" (phénoménologique) et l'idéalisme métaphysique qu'il combat : «avant tout, l'idéalisme phénoménologique ne nie pas l'existence effective du monde réel (et d'abord celle de la nature) comme s'il la prenait pour une apparence s'imposant, bien qu'à leur insu, à la pensée naturelle et à la pensée scientifique positive. II n'a qu'une seule tâche et une seule fonction [Leistung] : expliciter le sens de ce monde, précisément le sens par lequel il vaut pour tout un chacun comme existant effectivement, et de plein droit. Que le monde existe, que dans l'expérience continue convergeant sans cesse vers la concordance [Einstimmigkeif] universelle, il soit donné comme monde existant, voilà qui est parfaitement indubitables".

La Philosophie première (1923-1924) corrobore sans réserves cette absolue reconnaissance de «l'absolu» du Monde en phénoménologie : "l'existence du monde, déclare Husserl, est absolument hors de doute et cette indubitabilité est impliquée dans la perception du monde elle-même dans laquelle nous vivons continuellement. Quiconque se laissant égarer par des arguments sceptiques juge et croit que le monde en vérité n'existe pas, ou bien encore juge même simplement qu'il faut à chaque instant penser à cette possibilité, obéit à une motivation provenant d'arguments théoriques et sans doute même d'arguments d'ordre purement conceptuel et verbal; il méconnaît le contenu de sens de l'expérience du monde et l'inaltérable croyance au monde qu'elle recèle nonobstant tous les arguments de ce genre; c'est une croyance qui ne souffre en elle ni à côté d'elle le moindre soupçon ou la

8. "Postface à mes ldées directrices pour une phénoménologie pure" in La phénoménologie et les fondements des sciences, traduit par Dorian Tiffeneau, Paris : P.U.F., 1993, p. 198 [152-153]. 
moindre possibilité réelle qu'il en soit autrement 9 ». Retenons encore cette formidable sentence : "le monde est ce qu'il est, en soi et pour soi, que nous vivions ou que nous mourions, que nous le connaissions ou non 10 ». Saurait-on parler plus clairement?

L'article-synthèse de 1927 (art. «Phénoménologie» pour l'Encyclopedia Britannica comprend certes la phénoménologie dans l'enceinte herméneutique de l'idéalisme; mais cet idéalisme, donné pour transcendantal, vient sitôt désamorcer toute espèce d'exaltation «idéalisante" (à résonance volontariste et constructiviste, par exemple). La phénoménologie est "antimétaphysique» prétend Husserl, qui conclut : "En tant qu'eidétique, la phénoménologie est rationaliste, mais elle surmonte les limitations du rationalisme dogmatique en étudiant la subjectivité transcendantale, la conscience du Je et les objectivités qui valent pour la conscience. [...] Son idéalisme transcendantal sauvegarde le réalisme naturel et est prouvé non par des arguments aporétiques mais par les conséquences mêmes qu'implique le travail phénoménologique ${ }^{11}$ ».

Même les Méditations cartésiennes (1929), sommet incontesté de l'idéalisme husserlien, nous mettent en garde contre une lecture "absolutiste" de l'idéalisme transcendantal (qui perdrait de vue le corrélat de la transcendance). La distinction des mois psychologique et transcendantal a toujours et nécessairement besoin d'un horizon normatif de Transcendance, d'un véritable rapport au Monde, qui pour être réduit (dans la réflexion) n'en laisse pas d'opposer (dans l'intuition) l'immédiateté transcendantale du Moi à l'altérité réelle du Monde (comme "étrangeté irréductible», c'est-à-dire «non-moi»). D'où cette mise en garde, dès la première méditation : "Ce concept de transcendantal et son corrélatif, le concept de transcendant,

9. Philosophie première $\|$ : Théorie de la réduction phénoménologique, traduit par Arion L. Kelkel, Paris : P.U.F., 1990, 34e leçon, p. 75 [54].

10. Philosophie première I: Histoire critique des idées, traduit par Arion L. Kelkel, Paris : P.U.F., 1970, p. 308 [245].

11. "La phénoménologie (article pour l'Encyclopaedia Britannica), première version” in Notes sur Heidegger, traduit par Didier Franck, Paris : Minuit, 1993, p. 92. 
nous devrons les puiser exclusivement dans notre propre méditation philosophique. Remarquons à cet égard que, si le moi réduit n'est pas une partie du monde, de même, inversement, le monde et les objets du monde ne sont pas des parties réelles de mon moi12». Et Husserl d'ajouter : "L'être du monde (das Sein der Welt) est donc nécessairement "transcendant" à la conscience, même dans l'évidence originaire, et y reste nécessairement transcendant ${ }^{13}$ ". Force est de constater qu'au sein même de la réduction phénoménologique transcendantale, la Transcendance du Monde, en tant que condition irréductible de validation, vérification, concordance, se révèle éminemment garante de toute vérité "positive». L'idéalisme transcendantal n'est pas un anti-réalisme «dogmatique». II ne lutte pas contre le "dogme du monde" : il y croit. Ainsi que le comprend tout de bon Husserl : «ll est l'explicitation du sens [...] de la transcendance que l'expérience me donne réellement : celle de la Nature, de la Culture, du Monde [...] $]^{14 » \text {. }}$

Dans le même esprit, la conférence Phénoménologie et anthropologie (1931) déclare : «ll ne faut jamais perdre de vue que cette phénoménologie transcendantale ne fait rien d'autre que de questionner le monde, celui qui en tout temps est pour nous le monde effectif (qui vaut pour nous, se légitime pour nous, qui seul a sens pour nous) [... $]^{15}$ ". Le Monde, dont il est dit : "Son être est l'évidence constante, la présupposition constamment passée sous silence. Sa source est naturellement l'expérience universelle dans sa constante certitude

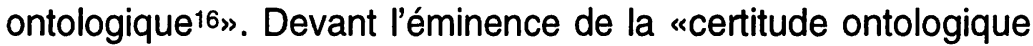
du monde", un constat s'impose : "Je ne peux, en fait, jamais la mettre en doute ou la nier17»".

12. Méditations cartésiennes, traduit par Gabrielle Peiffer et Emmanuel Lévinas, Paris : Vrin, 1980, § 11, p. 22.

13 Ibid., § 28, p. 52.

14. Ibid., § 41, p. 72.

15. "Phénoménologie et anthropologie" in Notes sur Heidegger, traduit par Didier Franck, Paris : Minuit, 1993, p. 71.

16. Ibid., p. 62.

17. Ibid. 
Dans cet important passage de la Krisis (1935-1936), Husserl retrace fort bien le pendant transcendantal de l'exigence "réaliste» transcendante : "Ce qui est d'avance, c'est le monde, toujours donné d'avance et hors de doute dans la certitude de l'être et la confirmation de soi-même. Quand bien même je ne l'ai pas "présupposé" comme sol, il n'en est pas moins pour moi, l'ego dans le cogito, un monde valide qui tire sa validation d'une auto-confirmation constante, avec tout ce qu'il est pour moi, qui dans le détail est parfois objectivement légitime, parfois non, y compris avec toutes les sciences, les arts, toutes les formes et les institutions sociales ou personnelles - aussi loin qu'il est, justement, le monde, qui est pour moi le monde véritable. II ne peut donc y avoir de réalisme plus fort, si par ce mot on n'entend rien de plus que : "je suis certain d'être un homme, qui vit dans ce monde, etc., et je n'ai pas là-dessus le moindre doute"18". II faudrait tout ignorer des concepts husserliens d'intuition, d'évidence, de certitude, et de leur place dans la théorie de la vérité, pour ne pas voir que ce "rien de plus» n'est qu'un euphémisme pour dire «rien de moins"! Dans le registre du savoir intuitif, la certitude correspond en effet au plus haut degré de l'évidence, dont l'idée coïncide avec la vérité. Husserl n'aura jamais renié l'enseignement de ses Recherches logiques. L'évidence originaire est le vécu de la vérité, l'aperception vivante (l'Idée vécue) de la concordance entre l'intention et son objet.

Notons encore que le texte posthume Expérience et Jugement (1938, par les soins de Landgrebe) s'ouvre précisément sur le thème du Monde comme sol universel de la croyance en tant que toujours déjà donné à toute expérience d'objets singuliers (Introduction, §7). On y retrouve toujours la même conviction : "L'être du monde dans sa totalité est ce qui va de soi, ce qui n'est jamais mis en doute, ce qui ne résulte pas d'une activité de jugement, mais qui constitue le présupposé de tout jugement. La conscience du monde est une conscience qui a pour mode la certitude de la croyance : elle

18. La crise des sciences européennes et la phénoménologie transcendantale, traduit par Gérard Granel, Paris : Gallimard, 1976, §55, p. 212-213. 
n'est pas obtenue par un acte exprès qui s'insérerait dans la continuité du vécu, comme acte de position de l'être, de saisie de l'étant, ou même comme l'acte du jugement prédicatif d'existence. Car tous ces actes présupposent déjà la conscience du monde dans la certitude de la croyance ${ }^{19}$ ”.

Enfin, il faut citer surtout la déclaration on ne peut plus claire du vieil Husserl à l'abbé-théologien Émile Baudin : "Kein gewöhnlicher "Realist" ist je so realistisch und so concret gewesen als ich, der phänomenologische "Idealist" (ein Wort, das ich übrigens nicht mehr gebrauche) 20 ". [Nul "réaliste» consacré ne fut jamais aussi réaliste et concret que moi, le phénoménologue «idéaliste» (un mot du reste, que je n'utilise plus).]

\section{Du sens normatif de la Thèse du Monde}

La découverte du monde comme dogme transcendantal a ceci de révélateur qu'elle fait apparaître le caractère essentiellement normatif de la croyance au Monde. L'absolu du Monde s'y révèle sous forme de nécessité téléologiquenormative, et non point comme une nécessité «matérielle», ou comme une nécessité de re, portant sur un contenu propositionnel déterminé. II s'ensuit que la thèse "épistémo-logique» du monde ne présente pas de contenu "onto-logique» irréfragable. L'eidos-monde tient lieu d'a priori corrélationnel universel dans le système transcendantal des intentionnalités objectivantes (tournées vers l'«au-dehors» indéfini) : d'où sa nécessité transcendantale, ou son invariance par variation.

Déterminé quant à sa forme, le dogme transcendantal du monde reste ouvert quant au sens d'être, au contenu, qu'il autorise. La "détermination» de la Thèse du Monde constitue l'idéal normatif de toute science, la tâche infinie de l'humanité savante. Nous n'en finirons jamais d'interroger le rapport du transcendant et du transcendantal dans l'horizon excédentaire

19. Expérience et Jugement, traduit par D. Souche, Paris : P.U.F., 1970, p. 34 [25].

20. “E. und M. Husserl an Baudin, 26. V./8. VI. 1934" in Edmund Husserl / Briefwechsel (Band VII) : Wissenschaftlerkorrespondenz, in Verbindung mit Elisabeth Schuhmann, herausgegeben von Karl Schuhmann, Dordrecht/Boston/London : Kluwer Academic Publishers, 1994, p. 16 [trad. par nos soins]. 
de l'Être. La métathéorie du couplage de la connaissance et du Monde représente à son tour une tâche infinie. Autrement dit, les sciences épistémologiques ne sauraient finir, car leurs sciences-objets n'ont pas de fin. Le sens d'être du Monde, comme celui des étants intramondains (soit le sens final de "l'étantité") comporte une dimension insondable. Présenter comme tâche infinie (donc humainement impossible...?) l'activité théorétique "déterminante", équivaut à reconnaître l'«indéterminité» effective de toute ontologie constituée. Or, cela signifie qu'aucune détermination "compréhensive" de l'Être, aucune ontologie philosophique, ni régionale ni fondamentale, ne sera jamais définitive.

L'indéterminité de l'ontologie se mesure d'abord à l'intimité du lien ontologie-langage. II n'est point d'ontologie qui ne soit tributaire d'un onto-logos, c'est donc dire, d'un raisonnement et de son langage. L'ontologie naît d'une rencontre : la rencontre du logos et de l'être dans l'analogie du savoir. Son indétermination permanente constitue le pendant "objectif» des limitations noétiques-transcendantales de l'«ontologos» constituant. Elle reflète l'amphibologie transcendantale du rapport de la fondation phénoménologique et du fondement transcendant. L'objectivation des formes et structures de l'être réclame sans cesse l'activité transcendantale de l'esprit objectivant. Porter l'être au langage, n'est jamais un processus univoque. À travers la réalité empirico-transcendantale du langage se constitue, en nous et pour nous, l'identité symbolique du donné antéprédicatif.

La question du rapport de l'ontologie au langage est également celle de son enracinement historial dans la Lebenswelt comme «lieu» de l'expérience donatrice originaire. Chez Husserl, c'est la théorie de l'intuition qui permet d'intégrer la double relation ontologie-langage/ontologie-Lebenswelt dans le lien Langage-Lebenswelt. «Le langage, écrivait Ricœur, conserve le savoir hors de l'intuition qui le justifie; cette dignité du langage est en même temps son péril21». On ne saurait 
rendre plus fidèlement la pensée de Husserl. L'appel à l'intuition peut assurément s'entendre, dans une certaine mesure, comme une exhortation à la re-découverte de ce qui est conservé dans le Langage, dans l'Histoire, dans la Culture, etc. Retourner aux choses elles-mêmes signifie d'abord remonter aux intuitions justifiantes, parcourir le chemin à rebours : des "sédiments de sens" à la Lebenswelt, en passant par la nécessaire Rückfrage. En dernière instance, le zurück zu den Sachen Selbst voudrait mesurer toute Weltanschauung à l'aune de la Lebenswelt : revoir toute "vision du monde" à la lumière du monde «vu » ou vécu.

\section{Attitude naturelle et précompréhension}

Avant toute objectivation théorétique du sujet humain, le phénomène de la subjectivité se trouve primordialement "donné» sous la forme d'une expérience intuitive plus ou moins définie, mais suffisamment profonde pour permettre le partage de l'existant en Moi et Non-moi. Cette possibilité de la vie antéthéorétique, suivant laquelle chacun dit "Je" et se distingue de tout Autre (d'autrui et du monde) est cela même qui deviendra, au regard de la raison théorétique, le problème de l'individuation. Ce qu'il faut comprendre, c'est que toute conception de l'identité personnelle, de quelque profondeur qu'elle soit (scientifique, philosophique, métaphysique, théologique), n'a de sens qu'en fonction de cette «précompréhension originaire", qu'il faut bien qualifier de naturelle puisqu'elle vient de soi-même à l'esprit.

La précompréhension de l'identité conditionne les divers schèmes représentationnels qui modulent, ici et maintenant, l'expérience naturelle que le Soi fait de soi-même. Dans la mesure où cette précompréhension sert de base et de principe au discours "autologique" de soi sur soi, elle conserve une fonction normative par-delà toute modélisation théorétique du Moi, ou Égologie. Une phénoménologie de la subjectivité devra d'abord «inventorier» ce qui est trouvé d'avance dans la spontanéité et l'être-là de la vie subjective-naturelle. Procéder à cet «inventaire» est précisément ce que Husserl appelle faire la 
description de l'attitude naturelle. S'il est évidemment impossible d'être exhaustif, il n'est pas non plus nécessaire de l'être. II suffit de parvenir ici à l'idée naïve-naturelle de la "mondanéité" dans sa détermination la plus générale, voire la plus vague. La "mondanéité" accompagne l'aperception spontanée de la vie dans sa manifestation primitive (de vie-d'avant-la-vie-quiobjective-la-vie). Aussi est-ce le point de départ obligé de tout retour réflexif sur cette forme de vie. Le sens formel-général de l'attitude naturelle ouvre l'espace heuristique où se déploie le génie des analyses objectivantes. L'idée d'une ontologie pure (a priori) de la nature trouve ici son sens comme système d'intentions "catégorielles" spécifiant le mode d'appréhension objectivo-naturel de l'être-là.

L'ontologie naïve-naturelle du monde de la vie se compose manifestement d'un univers d'objets et de personnes, avec toutes les interactions et les investissements de valeurs qu'une telle structure rend possible. Ici, les catégories ontothéorétiques des sciences positives n'interviennent pas de façon opératoire, pas plus d'ailleurs que l'ontologie philosophique dualiste du corps et de l'âme. On ne trouve rien de tel qu'un esprit "enveloppé" dans une chair-enveloppe. Ce n'est pas le dualisme fictif du corps-tombeau et de l'âmevivifiante qui donne à voir la différence ontologique, mais l'opposition empirico-transcendantale de la personne et de l'objet.

Beaucoup de philosophèmes transcendantaux, dont l'Idée de la pluralité des monades, s'annoncent déjà en quelque manière dans la précompréhension naturelle. Des interprétations malencontreuses de la réduction ont souvent laissé croire que l'attitude phénoménologique voulût déprécier, voire même discréditer le savoir obtenu dans l'attitude naturelle. Cela est foncièrement injuste et faux. La volonté de justifier l'expérience naturelle et le savoir positif, se révèle partout présente. En reconnaissant la valeur et la fécondité du concept normatif de monde naturel, c'est en vérité toute la sphère de l'expérience naturelle qui s'en trouve médiatement justifiée, sinon dans sa dernière facticité, du moins dans son principe, dans son droit. La vie, la vie positive, exige son droit, disait Husserl. Le droit de 
la vie positive se fait valoir de l'intérieur de la vie transcendantale. La vie du sujet solipsiste renvoie dialectiquement à la vie d'autrui comme à celle du monde. La téléologie exocentrique de la réduction n'est pas autre chose que l'histoire de sa sortie hors du domaine absolu de la cogitatio vers son au-delà objectif, dans l'unité aperceptive de l'esprit. En admettant qu'elle soit possible, la perfection de la réduction coïncide avec la double réfutation du scepticisme et du solipsisme (attendu que l'impossibilité d'une inclusion de tous les modes de conscience dans la conscience de soi montre la nécessité du passage de la conscience réduite à la conscience thétique). L'intention critique-transcendantale ne veut aucunement saper le savoir positif, mais en circonscrire le domaine, par la révélation de la dimension excédentaire de l'être, à commencer par l'immanence non naturalisable de la conscience transcendantale. En récusant la chute métaphysique du réalisme en matérialisme, la phénoménologie combat le rétrécissement anxiogène de l'être à l'étantité, elle refuse l'enfermement du naturel dans le naturalisme. La grande intuition phénoménologique, peut-être la plus grande, est qu'il se trouve un monde, un monde naturel, qui n'est pas, ou pas nécessairement, le monde du naturalisme, mais celui, plus proche et plus profond, de la Lebenswelt. En ce monde, et en ce monde seulement, le naturel trouve son acception absolue qui l'identifie au spontané, à l'originaire, à tout ce qui vient de soi-même. Le naturel précède le biologique et le psychologique qui tentent de le dominer. Le naturel est déjà le transcendantal. D'où le paradoxe de la subjectivité humaine, qui ne prouve pas la dualité métaphysique d'une composition, mais bien plutôt l'ambivalence ontologique d'une condition : l'humaine condition.

\section{Constitution et Création}

La résonance volontariste-constructiviste de l'allemand Konstitution, comme de l'ami français constitution, est sans doute la première responsable, sinon la cause occasionnelle, c'est-à-dire ici, linguistique, d'une malheureuse hypertrophie antiréaliste, et si j'ose dire "contre-nature», de la 
Konstitutionslehre. Pris dans le jeu langagier des rapprochements douteux et fausses amitiés, on se hasarde à transmuer tour à tour la constitution en formation, fabrication, production, construction... La fin - aberrante - de cette mutation artificielle serait d'égaler constitution du monde et Création $d u$ monde. Ainsi, entre l'origine concrète, soit le sujet transcendantal, et l'aboutissement métaphysique, le Sujet Créateur, la démesure est totale, et toute réconciliation paraît impossible, phénoménologiquement impossible. Soyons très clair : ni le sujet solipsiste transcendantal ni la Communauté intersubjective des Monades n'ont créé le Monde. II n'y a guère lieu, ce nous semble, de prêter à Husserl quelque secrète pensée de cette nature. Quoi qu'il en soit, si tel fut le cas, on aurait raison de prendre au pied la lettre les critiques "religieuse" et "existentielle» que Fink n'osa rapporter que sur un mode hypothétique (sans les diriger contre Husserl).

La critique d'inspiration religieuse s'élève contre la triple prétention qu'elle croit déceler dans la méthode de réduction : 1) celle de dépasser l'état de limitation et d'emprisonnement commun à tous les hommes; 2) de découvrir dans la profondeur dernière du Moi, la subjectivité créatrice du monde; 3) d'en faire le thème d'une connaissance rigoureusement scientifique. Voilà qui semble, constate Fink, "un parfait orgueil, une outrecuidance et une excentricité, une hybris sans pareille; exprimée d'un point de vue religieux, c'est l'arrogance de la créature se mettant elle-même à la place de Dieu22». De fait, l'activité constituante du sujet transcendantal œuvre toujours déjà à l'intérieur du don de l'Être. La parole constituante de l'Homme n'est assurément pas la Parole efficace du Dieu à qui il suffit de dire pour que les choses arrivent, d'ordonner pour qu'elles existent (Ps 33, 9).

La critique existentielle, qui peut paraître supérieure, en cela même qu'elle prétend se passer de la foi, soulève à son tour d'inquiétants soupçons quant à la propension

22. Eugen Fink, Sixième méditation cartésienne, trad. par Nathalie Depraz, Grenoble : Editions Jérome Millon, 1994, p. 168 [122-123]. 
constructiviste du subjectivisme moderne. Elle s'inscrit dans la foulée des critiques "postmodernes" qui dénoncent la déperdition du subjectivisme transcendantal en idéalisme constructif. Parmi les soupçons signalés par Fink, relevons cette interrogation : "ce "titanisme" téméraire qui s'atteste dans la construction du sujet transcendantal n'est-il finalement que du désoeuvrement spirituel, une chimère intellectuelle bien légère qui est le fait d'une vie rendue étrangère aux réalités effectives proprement dites, menaçantes et bouleversantes de l'existence humaine (la mort, le destin, la faute et autres "choses ultimes")23?". Voilà une bonne question... laissée sans réponse.

Tout porte à penser que la phénoménologie constitutive répugne à toute interprétation métaphysique constructiviste. Non seulement Husserl rejette-t-il la notion idéaliste absolue de construction d'objets, mais en outre, celle de construction de concepts. L'une et l'autre lui paraissent également absurde : «si par concept on entend précisément l'essence [...] dans ce cas l'expression de produits psychiques est un non-sens, également celle de construction de concepts, pour autant qu'on doive l'entendre en un sens rigoureux et authentique24». Et Husserl de nuancer : "ce n'est pas l'essence mais la conscience de l'essence qui est un produit de l'esprit25". Suivant la doctrine husserlienne de la signification, l'aperception d'essence, comme l'aperception de vérité, sont des intuitions «intellectuelles" ("catégoriales») qui attestent transcendantalement l'expérience d'une donation originaire. Nous ne produisons pas les essences; nous n'inventons pas la vérité. Le sens de l'être préexiste (en même temps qu'il prédispose) à la recherche du sens. Si l'être, dans son indétermination phénoménologique première, n'annonçait pas déjà un sens, toute objectivation théorétique serait vaine, mais aussi, toute espèce de "monstration» esthétique (poétique, musicale, chorégraphique, picturale, etc.). II n'y aurait alors rien à dire, ni à laisser entendre, rien qui ne puisse être porté au Langage sous quelque forme que ce soit, pas même une émotion de la 23. Ibid., p. 168 [123].

24. Idées I, p. 74-75 [41].

25. Idées I, p. 76 [43]. 
«bête humaine», car aucune émotion n'est absurde ou in-signifiante, comme chacun sait.

L'anticonstructivisme de Husserl dépasse la simple dénonciation de principe. II se confirme dans l'exercice concret de la phénoménologie constitutive. Les leçons si fondamentales de 1907 sur la constitution de la chose et de l'espace ne laisseront ici aucun doute. Elles s'inscrivent expressément dans le cadre d'une phénoménologie de l'expérience et se veulent une première élucidation de l'essence de la donation empirique, une exégèse de ses niveaux inférieurs : les intuitions présentifiantes préscientifiques de la donnée-de-chose. Dès l'introduction, Husserl oppose sa "phénoménologie de l'expérience» à la "théorie de l'expérience» au sens néo-kantien de Cohen et des membres de l'École de Marbourg (dont Cassirer). II refuse d'infléchir son concept de constitution en ce sens constructiviste, car, soutient-il, «rien ne doit être expliqué ni fondé en ce sens'26».

\section{Pour une compréhension «herméneutique» de la constitution}

II appert insuffisant de s'en tenir à une idée seulement négative, anticonstructiviste, de la constitution. Aussi devonsnous indiquer l'acception "positive» à laquelle nous voulons nous rallier. Cette acception se fonde sur une interprétation "représentationnelle», ou mieux, «herméneutique», du concept de constitution. L'idée n'est pas inédite : elle se pourrait même réclamer du «vrai» Husserl, si l'on en croit sa déclaration à Hocking du 25 janvier 1903. À notre connaissance, Walter Biemel fut le premier à prendre cette déclaration suffisamment au sérieux pour croire à la nature essentiellement «herméneutique» de la Konstitutionslehre. "L'expression qui revient tant de fois, écrit Husserl, que des "objets" se "constituent" dans un acte, signifie toujours la propriété de l'acte de rendre

26. Chose et espace, traduit par Jean-François Lavigne, Paris : P.U.F., 1989, p. 24 [4].

27. là-dessus, voir la conférence fondamentale de Biemel sur "Les phases décisives dans le développement de la philosophie de Husserl", in Husserl (Congrès de Royaumont), Paris : Minuit, 1959, p. 32-62. 
représentable l'objet ; il ne s'agit pas de "constituer" au sens propre27". Poser le problème de la constitution, c'est tenter d'expliciter la genèse subjective de l'objectif, le devenirintentionnel de l'objet, sa présentation ou présentification comme présence-à-la-conscience (noème). On ne le dira jamais suffisamment : le concept phénoménologique de constitution ne doit rien à l'idéalisme allemand; il s'inspire, quant à l'histoire s'entend, de l'empirisme anglais. De l'aveu même de Husserl, c'est Hume qui fut le premier à saisir le problème de la constitution ${ }^{28}$ (quoique son idée de la genèse subjective ne fût point sans reproches).

Sans verser dans le scepticisme humien, le point de vue génétique radical de la phénoménologie consacre le statut rigoureusement interprétatif de toute constitution, comme l'a bien vu Rudolf Bœhm à l'occasion d'un rapprochement, inattendu mais heureux, entre Husserl et Nietzsche : «Formellement, écrit Bœhm, toute constitution, au sens phénoménologique, est interprétation, dans la mesure où toute constitution est, conformément à l'usage courant de ce terme, constitution de "quelque chose" en "quelque chose"29». La konstitutionslehre développe une conception générale de la représentation fondée sur la vision génétique-transcendantale des sens, modes et attributions d' "existence" (d' "être»). Les actes constituants comprennent donc l'ensemble des processus intentionnels d'accession à la représentation. Les problèmes constitutifs définis sur une certaine classe d' "objets" sont des problèmes de genèse intentionnelle. L'analytique constitutive d'une région-objet consiste en une analyse plurielle des modes-types de donation de ses individus caractéristiques. L'analytique constitutive peut s'étendre à toute espèce d' «objectité» qui présente quelque Thèse ou "Position d'existence" en quelque sens que ce soit, y

28. sur cette reconnaissance de dette, voir Logique formelle et logique transcendantale, § 100. Remarques historico-critiques sur le développement de la philosophie transcendantale et en particulier sur la problématique transcendantale de la logique formelle; aussi Philosophie première I, Troisième Section, Chapitre II, Le positivisme de Hume - à la fois accomplissement du scepticisme et pas préliminaire décisif conduisant à une science transcendantale fondamentale.

29. Rudolf Bœhm, "Deux points de vue : Husserl et Nietzsche" in Archivio di Filosofia, no. 3, 1962, p. 173. 
compris les innombrables "transcendances dans l'immanence». D'où, à rebours, l'universalité du problème de la genèse, dont la signification "herméneutique" paraît plus évidente. Rapportée à l'ensemble du domaine objectal (mondain), la multiplicité des exégèses constitutives compose -idéalement- une vaste herméneutique de la facticité.

L'universalité du problème constitutif traduit l'universalité de I'herméneutique. En un sens - qu'il faut dissocier d'un perspectivisme niais et béat - tout est affaire d'interprétation. On ne sort d'une interprétation que pour aboutir dans une autre. Qu'on y prenne un malin plaisir ou qu'on s'en repente, la sémantique maudite a toujours le dernier mot dans la Babel des hommes. Hors de la Nuance, point d'équilibre au pays de la Confusion. La Nuance qui nous enchaîne... nous entraîne... nous libère. Ce miracle à visage humain, nous l'appelons Philosophie. La Philosophie, ou l'Art de la Nuance Raisonnable.

\section{Le paradoxe}

\section{du «paradoxe de la subjectivité humaine»}

Si le projet husserlien de fondation transcendantale de la transcendance n'est pas un programme de réduction au sens positiviste, c'est d'abord parce qu'il cherche à maintenir l'autonomie respective des deux ordres topologiques fondatifs, à savoir, suivant l'heureuse appellation de Jacques English : la topologie immanente de la fondation et la topologie transcendante du fondement 30 . La distinctio phaenomenologica qui inaugure le projet phénoménologique suppose en effet la possibilité d'une séparation thématique des topologies immanente et transcendante (donc aussi une certaine autonomie de la raison transcendantale par rapport à la rationalité positive). Par contre, elle ne rompt pas la réciprocité "apparente», c'est-à-dire phénoménologique, du transcendant et du transcendantal, comme l'atteste diversement quelques-uns des plus grands thèmes (méta)phénoménologiques : introjection transcendantale de la mondanéité, allocalisation mondaine de la vie transcendantale, immixtion du savoir apriorique dans la science

30. Sur cette terminologie, qui parle d'elle-même, voir la préface de Jacques English à sa traduction française des Grundprobleme der Phänomenologie op. cit. 
empirique, efficience de la "technique" (sur le Moi comme sur le Monde), dépendance psycho-physique (causale et/ou motivationnelle).

Il importe de réaliser qu'une référence immanente à la positivité continue sans cesse de se faire valoir au sein même de l'attitude transcendantale radicale. L'épochè phénoménologique n'abolit pas la positivité, mais la donne à voir en tant que telle, c'est donc dire qu'elle la limite, la circonscrit. Toutefois, ce n'est qu'au prix d'un va-et-vient continuel entre conscience naturelle et conscience réduite que leur domaine respectif se donne à penser. Or il s'agit là d'une situation hautement problématique, car cela signifie que la légalité de la topologie transcendante du fondement ne saurait se justifier complètement par les seuls moyens de la fondation transcendantale. D'un point de vue transcendantal, le passage de la topologie immanente à la topologie transcendante semble comporter un saut irrationnel : une foi en la Transcendance, peut-on dire. Ainsi, la positivité naturelle apparaît toujours posée sur fond d'une naïveté originelle insurmontable. Au regard de la conscience naturelle, la Nature est toujours "plus" que le dogme transcendantal reconnu dans l'attitude de réduction. Ce «plus" pèse sur le cœur de la phénoménologie telle l'épée sur la tête de Damoclès, car il menace l'existence même, sinon l'universalité, de la fondation transcendantale. Ou bien le Transcendant se déduit nécessairement du Transcendantal (par je ne sais quelle déduction eidétique), et par conséquent, la Réduction comme suspension totale des Thèses d'existence se révèle intenable; ou bien une pareille déduction est impossible, et dès lors l'«universalité même" de la fondation transcendantale se voit mise en échec.

Le refus d'abandonner la topologie transcendante du fondement, malgré l'indéniable impasse de toute déduction eidético-transcendantale de la Transcendance, atteste bien l'authenticité de la conviction réaliste de Husserl : sa «foi» en la Transcendance est somme toute inébranlable. C'est précisément parce que Husserl prend très au sérieux la topologie transcendante du fondement que le paradoxe de la subjectivité humaine (tout à la fois sujet dans le monde et objet du monde) se présente comme un problème phénoménologique crucial. Au 
fond, le paradoxe du sujet-objet (de l'objet-sujet) ne fait que traduire sur le plan anthropologique l'«impossibilité" (transcendantale) de (ré)concilier la topologie immanente de la fondation avec la topologie transcendante du fondement. Cet échec, s'il est inévitable, est aussi l'échec de l'épochè phénoménologique universelle, comme le reconnaissait d'ailleurs le dernier Husserl. Dans la Krisis, il écrit : «Si le paradoxe [...] [de la subjectivité humaine] ne pouvait être résolu, cela signifierait que d'une façon générale il n'est pas possible d'accomplir une épochè universelle et radicale, et ce en vue d'une science qui lui soit attachée rigoureusement ${ }^{31}$ ».

La seule solution phénoménologiquement envisageable consisterait à "résoudre" (donc à "dissoudre») le paradoxe de la subjectivité humaine par une analytique généticointentionnelle de ces différentes couches constitutives (par "déconstruction" pourrait-on dire). Tel était l'ambitieux dessein des Ideen II. Or, il appert à nos yeux que cette "solution" escamote le mystère profond de la causalité physio-psychique : "On dit bien sûr, rapporte Husserl, qu'une causalité physio-psychique est une énigme. Mais une telle "énigme" n'appartientelle pas à l'essence de toute causation? Cela veut dire alors, conclut-il, qu'il n'y a pas d'énigme du tout32». À dire vrai, nous ne pouvons voir en cet argument autre chose qu'un sophisme essentialiste, aussi peu convaincant qu'une "preuve» ontologique de l'existence de Dieu. D'ailleurs, il n'est pas interdit de penser que Husserl lui-même n'en fut pas convaincu, puisque l'auteur de la Krisis - pourtant si préoccupé par ce problème - ne mentionne même plus cet argument. Quoi qu'il en soit, tout porte à penser qu'on ne saurait venir à bout du singulier paradoxe de la subjecti(vi)té humaine.

\section{Éric Paquette Département de philosophie Collège Édouard-Montpetit}

31. "Le paradoxe de la subjectivité humaine" in La crise des sciences européennes et la phénoménologie transcendantale, traduit par Gérard Granel, Paris : Gallimard, 1976, § 53, p. 205.

32. Recherches phénoménologiques pour la constitution, traduit par Éliane Escoubas, Paris : P.U.F., 1982, § 60, p. 352 [259-260]. 